\title{
Numerical simulation for unsteady anisotropic-diffusion convection equation of spatially variable coefficients and incompressible flow
}

\author{
Mohammad Ivan Azis
}

\begin{abstract}
The anisotropic-diffusion convection equation of spatially variable coefficients which is relevant for functionally graded media is discussed in this paper to find numerical solutions by using a combined Laplace transform and boundary element method. The variable coefficients equation is transformed to a constant coefficients equation. The constant coefficients equation is then Laplace-transformed so that the time variable vanishes. The Laplace-transformed equation is consequently written in a pure boundary integral equation which involves a time-free fundamental solution. The boundary integral equation is therefore employed to find numerical solutions using a standard boundary element method. Finally the results obtained are inversely transformed numerically using the Stehfest formula to get solutions in the time variable. The combined Laplace transform and boundary element method is easy to be implemented and accurate for solving unsteady problems of anisotropic functionally graded media governed by the diffusion convection equation.
\end{abstract}

Keywords. Functionally graded materials, variable coefficients, anisotropic, unsteady, diffusion-convection

\section{Introduction}

In the last decade investigations on the diffusion-convection equation had been done for finding its numerical solutions. The investigations can be classified according to the anisotropy and inhomogeneity of the media under consideration. For example, Wu et al. [34], Hernandez-Martinez et al. [12], Wang et al. [33] and Fendoğlu et al. [7] had been working on problems of isotropic diffusion and homogeneous media, Yoshida and Nagaoka [35], Meenal and Eldho [19], Azis [3] (for Helmholtz type governing equation) studied problems of anisotropic diffusion but homogeneous media. Rap et al. [23], Ravnik and Škerget [25, 26], Li et al. [18] and Pettres and Lacerda [22] considered the case of isotropic diffusion and variable coefficients (inhomogeneous media). Recently Azis and co-workers had been working on steady state problems of anisotropic inhomogeneous media for several types of governing equations, for examples [5, 32] for the modified Helmholtz equation, [4, 14, 24, 30, 27, 11, 17] for the diffusion convection reaction equation, $[29,8,13,16]$ for the Laplace type equation, $[10,2,20,21,15]$ for the Helmholtz equation.

Received date: December 29, 2020; Published online: November 10, 2021.

2010 Mathematics Subject Classification. 65M38, 35K51, 44A10, 35N10.

Corresponding author: Mohammad Ivan Azis. 
Referred to the Cartesian frame $O x_{1} x_{2}$ we will consider initial boundary value problems governed by an unsteady anisotropic-diffusion convection equation with incompressible flow and variable coefficients of the form

$$
\frac{\partial}{\partial x_{i}}\left[d_{i j}(\mathbf{x}) \frac{\partial c(\mathbf{x}, t)}{\partial x_{j}}\right]-\frac{\partial}{\partial x_{i}}\left[v_{i}(\mathbf{x}) c(\mathbf{x}, t)\right]=\alpha(\mathbf{x}) \frac{\partial c(\mathbf{x}, t)}{\partial t}
$$

The coefficient $\left[d_{i j}\right](i, j=1,2)$ is a real positive definite symmetrical matrix. Also, in (1.1) the summation convention for repeated indices holds, so that explicitly (1.1) can be respectively written as

$$
\begin{aligned}
& \frac{\partial}{\partial x_{1}}\left(d_{11} \frac{\partial c}{\partial x_{1}}\right)+\frac{\partial}{\partial x_{1}}\left(d_{12} \frac{\partial c}{\partial x_{2}}\right)+\frac{\partial}{\partial x_{2}}\left(d_{12} \frac{\partial c}{\partial x_{1}}\right)+\frac{\partial}{\partial x_{2}}\left(d_{22} \frac{\partial c}{\partial x_{2}}\right) \\
& -\frac{\partial}{\partial x_{1}}\left(v_{1} c\right)-\frac{\partial}{\partial x_{2}}\left(v_{2} c\right)=\alpha \frac{\partial c}{\partial t}
\end{aligned}
$$

Equation (1.1) is used to model unsteady diffusion convection process in anisotropic and inhomogeneous (functionally graded) materials. Among the physical phenomena of applications include pollutant transport and heat transfer.

Nowadays functionally graded materials (FGMs) have become an important issue, and numerous studies on this issue for a variety of applications have been reported. Authors commonly define an FGM as an inhomogeneous material having a specific property such as thermal conductivity, hardness, toughness, ductility, corrosion resistance, etc. that changes spatially in a continuous fashion. Therefore equation (1.1) is relevant for FGMs.

Equation (1.1) applies for unsteady problems of anisotropic and inhomogeneous therefore provides a wider class of problems. It covers problems of isotropic and homogeneous media as special cases which occur respectively when $d_{11}=d_{22}, d_{12}=0$ and the coefficients $d_{i j}, v_{i}$ and $\alpha$ are constant.

Zoppou and Knight [36] had been working on finding the analytical solution to the unsteady orthotropic diffusion-convection equation with spatially variable coefficients. The equation considered is almost similar to equation (1.1) but with limitation $d_{11} \neq d_{22}, d_{12}=0$. This paper is intended to extend the recently published works on anisotropic diffusion convection equation with variable coefficients $[31,6,9,28,1]$ from the steady state to unsteady state equation.

\section{The initial boundary value problem}

Given the coefficients $d_{i j}(\mathbf{x}), v_{i}(\mathbf{x}), \alpha(\mathbf{x})$ solutions $c(\mathbf{x}, t)$ and its derivatives to (1.1) are sought which are valid for time interval $t \geq 0$ and in a region $\Omega$ in $R^{2}$ with boundary $\partial \Omega$ which consists of a finite number of piecewise smooth curves. On $\partial \Omega_{1}$ the dependent variable $c(\mathbf{x}, t)$ is specified, and

$$
P(\mathbf{x}, t)=d_{i j}(\mathbf{x}) \frac{\partial c(\mathbf{x}, t)}{\partial x_{i}} n_{j}
$$

is specified on $\partial \Omega_{2}$ where $\partial \Omega=\partial \Omega_{1} \cup \partial \Omega_{2}$ and $\mathbf{n}=\left(n_{1}, n_{2}\right)$ denotes the outward pointing normal to $\partial \Omega$. The initial condition is taken to be

$$
c(\mathbf{x}, 0)=0
$$

The method of solution will be to transform the variable coefficient equation (1.1) to a constant coefficient equation, and then taking a Laplace transform of the constant coefficient equation, and 
to obtain a boundary integral equation in the Laplace transform variable $s$. The boundary integral equation is then solved using a standard boundary element method (BEM). An inverse Laplace transform is applied to get the solution $c$ and its derivatives for all $(\mathbf{x}, t)$ in the domains. The inverse Laplace transform is implemented numerically using the Stehfest formula. The analysis is specially relevant to an anisotropic medium but it equally applies to isotropic media. For isotropy, the coefficients in (1.1) take the form $d_{11}=d_{22}$ and $d_{12}=0$ and use of these equations in the following analysis immediately yields the corresponding results for an isotropic medium.

The analysis in this paper is purely mathematical, the main aim is to derive a BEM for finding the numerical solutions to problems governed by equation (1.1).

\section{The boundary integral equation}

We restrict the coefficients $d_{i j}, v_{i}, \alpha$ to be of the form

$$
\begin{aligned}
d_{i j}(\mathbf{x}) & =\hat{d}_{i j} g(\mathbf{x}) \\
v_{i}(\mathbf{x}) & =\hat{v}_{i} g(\mathbf{x}) \\
\alpha(\mathbf{x}) & =\hat{\alpha} g(\mathbf{x})
\end{aligned}
$$

where $g(\mathbf{x})$ is a differentiable function and $\hat{d}_{i j}, \hat{v}_{i}, \hat{\alpha}$ are constants. Further we assume that the coefficients $d_{i j}(\mathbf{x}), v_{i}(\mathbf{x})$ and $\alpha(\mathbf{x})$ are exponentially graded by taking $g(\mathbf{x})$ as an exponential function

$$
g(\mathbf{x})=\left[\exp \left(\beta_{0}+\beta_{i} x_{i}\right)\right]^{2}
$$

where $\beta_{0}$ and $\beta_{i}$ are constants. Therefore if

$$
\hat{d}_{i j} \beta_{i} \beta_{j}-\lambda=0
$$

then (3.4) satisfies

$$
\hat{d}_{i j} \frac{\partial^{2} g^{1 / 2}}{\partial x_{i} \partial x_{j}}-\lambda g^{1 / 2}=0
$$

Substitution of (3.1)-(3.3) into (1.1) gives

$$
\hat{d}_{i j} \frac{\partial}{\partial x_{i}}\left(g \frac{\partial c}{\partial x_{j}}\right)-\hat{v}_{i} g \frac{\partial c}{\partial x_{i}}=\hat{\alpha} g \frac{\partial c}{\partial t}
$$

Assume

$$
c(\mathbf{x}, t)=g^{-1 / 2}(\mathbf{x}) \psi(\mathbf{x}, t)
$$

therefore substitution of (3.1) and (3.8) into (2.1) gives

$$
P(\mathbf{x}, t)=-P_{g}(\mathbf{x}) \psi(\mathbf{x}, t)+g^{1 / 2}(\mathbf{x}) P_{\psi}(\mathbf{x}, t)
$$

where

$$
P_{g}(\mathbf{x}, t)=\hat{d}_{i j} \frac{\partial g^{1 / 2}(\mathbf{x})}{\partial x_{j}} n_{i} \quad P_{\psi}(\mathbf{x}, t)=\hat{d}_{i j} \frac{\partial \psi(\mathbf{x}, t)}{\partial x_{j}} n_{i}
$$

And equation (3.7) can be written as 


$$
\begin{gathered}
\hat{d}_{i j} \frac{\partial}{\partial x_{i}}\left[g \frac{\partial\left(g^{-1 / 2} \psi\right)}{\partial x_{j}}\right]-\hat{v}_{i} g \frac{\partial\left(g^{-1 / 2} \psi\right)}{\partial x_{i}}=\hat{\alpha} g \frac{\partial\left(g^{-1 / 2} \psi\right)}{\partial t} \\
\hat{d}_{i j} \frac{\partial}{\partial x_{i}}\left[g\left(g^{-1 / 2} \frac{\partial \psi}{\partial x_{j}}+\psi \frac{\partial g^{-1 / 2}}{\partial x_{j}}\right)\right]-\hat{v}_{i} g\left(g^{-1 / 2} \frac{\partial \psi}{\partial x_{i}}+\psi \frac{\partial g^{-1 / 2}}{\partial x_{i}}\right)=\hat{\alpha} g\left(g^{-1 / 2} \frac{\partial \psi}{\partial t}\right) \\
\hat{d}_{i j} \frac{\partial}{\partial x_{i}}\left(g^{1 / 2} \frac{\partial \psi}{\partial x_{j}}+g \psi \frac{\partial g^{-1 / 2}}{\partial x_{j}}\right)-\hat{v}_{i}\left(g^{1 / 2} \frac{\partial \psi}{\partial x_{i}}+g \psi \frac{\partial g^{-1 / 2}}{\partial x_{i}}\right)=\hat{\alpha} g^{1 / 2} \frac{\partial \psi}{\partial t}
\end{gathered}
$$

Use of the identity

$$
\frac{\partial g^{-1 / 2}}{\partial x_{i}}=-g^{-1} \frac{\partial g^{1 / 2}}{\partial x_{i}}
$$

implies

$$
\begin{gathered}
\hat{d}_{i j} \frac{\partial}{\partial x_{i}}\left(g^{1 / 2} \frac{\partial \psi}{\partial x_{j}}-\psi \frac{\partial g^{1 / 2}}{\partial x_{j}}\right)-\hat{v}_{i}\left(g^{1 / 2} \frac{\partial \psi}{\partial x_{i}}-\psi \frac{\partial g^{1 / 2}}{\partial x_{i}}\right)=\hat{\alpha} g^{1 / 2} \frac{\partial \psi}{\partial t} \\
g^{1 / 2}\left(\hat{d}_{i j} \frac{\partial^{2} \psi}{\partial x_{i} \partial x_{j}}-\hat{v}_{i} \frac{\partial \psi}{\partial x_{j}}\right)-\psi\left(\hat{d}_{i j} \frac{\partial^{2} g^{1 / 2}}{\partial x_{i} \partial x_{j}}-\hat{v}_{i} \frac{\partial g^{1 / 2}}{\partial x_{i}}\right) \\
+\left(\hat{d}_{i j} \frac{\partial \psi}{\partial x_{j}} \frac{\partial g^{1 / 2}}{\partial x_{i}}-\hat{d}_{i j} \frac{\partial \psi}{\partial x_{j}} \frac{\partial g^{1 / 2}}{\partial x_{i}}\right)=\hat{\alpha} g^{1 / 2} \frac{\partial \psi}{\partial t}
\end{gathered}
$$

For incompressible flow

$$
\frac{\partial v_{i}(\mathbf{x})}{\partial x_{i}}=2 g^{1 / 2}(\mathbf{x}) \hat{v}_{i} \frac{\partial g^{1 / 2}(\mathbf{x})}{\partial x_{i}}=0
$$

that is

$$
\hat{v}_{i} \frac{\partial g^{1 / 2}(\mathbf{x})}{\partial x_{i}}=0
$$

Thus (3.10) becomes

$$
g^{1 / 2}\left(\hat{d}_{i j} \frac{\partial^{2} \psi}{\partial x_{i} \partial x_{j}}-\hat{v}_{i} \frac{\partial \psi}{\partial x_{i}}\right)-\psi \hat{d}_{i j} \frac{\partial^{2} g^{1 / 2}}{\partial x_{i} \partial x_{j}}=\hat{\alpha} g^{1 / 2} \frac{\partial \psi}{\partial t}
$$

Equation (3.6) then implies

$$
\hat{d}_{i j} \frac{\partial^{2} \psi}{\partial x_{i} \partial x_{j}}-\hat{v}_{i} \frac{\partial \psi}{\partial x_{i}}-\lambda \psi=\hat{\alpha} \frac{\partial \psi}{\partial t}
$$

Taking a Laplace transform of (3.8), (3.9), (3.11) and applying the initial condition (2.2) we obtain

$$
\psi^{*}(\mathbf{x}, s)=g^{1 / 2}(\mathbf{x}) c^{*}(\mathbf{x}, s)
$$




$$
\begin{gathered}
P_{\psi^{*}}(\mathbf{x}, s)=\left[P^{*}(\mathbf{x}, s)+P_{g}(\mathbf{x}) \psi^{*}(\mathbf{x}, s)\right] g^{-1 / 2}(\mathbf{x}) \\
\hat{d}_{i j} \frac{\partial^{2} \psi^{*}}{\partial x_{i} \partial x_{j}}-\hat{v}_{i} \frac{\partial \psi^{*}}{\partial x_{i}}-(\lambda+s \hat{\alpha}) \psi^{*}=0
\end{gathered}
$$

where $s$ is the variable of the Laplace-transformed domain.

By using Gauss divergence theorem, equation (3.14) can be transformed into a boundary integral equation

$$
\begin{aligned}
\eta(\boldsymbol{\xi}) \psi^{*}(\boldsymbol{\xi}, s)= & \int_{\partial \Omega}\left\{P_{\psi^{*}}(\mathbf{x}, s) \Phi(\mathbf{x}, \boldsymbol{\xi})-\left[P_{v}(\mathbf{x}) \Phi(\mathbf{x}, \boldsymbol{\xi})\right.\right. \\
& \left.+\Gamma(\mathbf{x}, \boldsymbol{\xi})] \psi^{*}(\mathbf{x}, s)\right\} d S(\mathbf{x})
\end{aligned}
$$

where

$$
P_{v}(\mathbf{x})=\hat{v}_{i} n_{i}(\mathbf{x})
$$

For 2-D problems the fundamental solutions $\Phi(\mathbf{x}, \boldsymbol{\xi})$ and $\Gamma(\mathbf{x}, \boldsymbol{\xi})$ are given as

$$
\begin{aligned}
\Phi(\mathbf{x}, \boldsymbol{\xi}) & =\frac{\rho_{i}}{2 \pi D} \exp \left(-\frac{\dot{\mathbf{v}} \cdot \dot{\mathbf{R}}}{2 D}\right) K_{0}(\dot{\mu} \dot{R}) \\
\Gamma(\mathbf{x}, \boldsymbol{\xi}) & =\hat{d}_{i j} \frac{\partial \Phi(\mathbf{x}, \boldsymbol{\xi})}{\partial x_{j}} n_{i}
\end{aligned}
$$

where

$$
\begin{gathered}
\dot{\mu}=\sqrt{(\dot{v} / 2 D)^{2}+[(\lambda+s \hat{\alpha}) / D]} \\
D=\left[\hat{d}_{11}+2 \hat{d}_{12} \rho_{r}+\hat{d}_{22}\left(\rho_{r}^{2}+\rho_{i}^{2}\right)\right] / 2 \\
\dot{\mathbf{R}}=\dot{\mathbf{x}}-\dot{\boldsymbol{\xi}} \\
\dot{\mathbf{x}}=\left(x_{1}+\rho_{r} x_{2}, \rho_{i} x_{2}\right) \\
\dot{\boldsymbol{\xi}}=\left(\xi_{1}+\rho_{r} \xi_{2}, \rho_{i} \xi_{2}\right) \\
\dot{\mathbf{v}}=\left(\hat{v}_{1}+\rho_{r} \hat{v}_{2}, \rho_{i} \hat{v}_{2}\right) \\
\dot{R}=\sqrt{\left(x_{1}+\rho_{r} x_{2}-\xi_{1}-\rho_{r} \xi_{2}\right)^{2}+\left(\rho_{i} x_{2}-\rho_{i} \xi_{2}\right)^{2}} \\
\dot{v}=\sqrt{\left(\hat{v}_{1}+\rho_{r} \hat{v}_{2}\right)^{2}+\left(\rho_{i} \hat{v}_{2}\right)^{2}}
\end{gathered}
$$

where $\rho_{r}$ and $\rho_{i}$ are respectively the real and the positive imaginary parts of the complex root $\rho$ of the quadratic equation

$$
\hat{d}_{11}+2 \hat{d}_{12} \rho+\hat{d}_{22} \rho^{2}=0
$$

and $K_{0}$ is the modified Bessel function. Use of (3.12) and (3.13) in (3.15) yields

$$
\eta g^{1 / 2} c^{*}=\int_{\partial \Omega}\left\{\left(g^{-1 / 2} \Phi\right) P^{*}+\left[\left(P_{g}-P_{v} g^{1 / 2}\right) \Phi-g^{1 / 2} \Gamma\right] c^{*}\right\} d S
$$

Equation (3.18) provides a boundary integral equation for determining the numerical solutions of $c^{*}$ and its derivatives $\partial c^{*} / \partial x_{1}$ and $\partial c^{*} / \partial x_{2}$ at all points of $\Omega$.

Knowing the solutions $c^{*}(\mathbf{x}, s)$ and its derivatives $\partial c^{*} / \partial x_{1}$ and $\partial c^{*} / \partial x_{2}$ which are obtained from (3.18), the numerical Laplace transform inversion technique using the Stehfest formula is 
Table 1: Values of $V_{m}$ of the Stehfest formula for $N=4,6,8,10$

\begin{tabular}{|c|c|c|c|c|}
\hline$V_{m}$ & $N=4$ & $N=6$ & $N=8$ & $N=10$ \\
\hline \hline$V_{1}$ & -2 & 1 & $-1 / 3$ & 0.083333333333333 \\
\hline$V_{2}$ & 26 & -49 & $145 / 3$ & -32.083333333333336 \\
\hline$V_{3}$ & -48 & 366 & -906 & 1279 \\
\hline$V_{4}$ & 24 & -858 & $16394 / 3$ & -15623.666666666666 \\
\hline$V_{5}$ & & 810 & $-43130 / 3$ & 84244.1666666666 \\
\hline$V_{6}$ & & -270 & 18730 & -236957.5 \\
\hline$V_{7}$ & & & $-35840 / 3$ & 375911.6666666666 \\
\hline$V_{8}$ & & & $8960 / 3$ & -340071.6666666666 \\
\hline$V_{9}$ & & & & 164062.5 \\
\hline$V_{10}$ & & & & -32812.5 \\
\hline
\end{tabular}

then employed to find the values of $c(\mathbf{x}, t)$ and its derivatives $\partial c / \partial x_{1}$ and $\partial c / \partial x_{2}$. The Stehfest formula is

$$
\begin{aligned}
c(\mathbf{x}, t) & \simeq \frac{\ln 2}{t} \sum_{m=1}^{N} V_{m} c^{*}\left(\mathbf{x}, s_{m}\right) \\
\frac{\partial c(\mathbf{x}, t)}{\partial x_{1}} & \simeq \frac{\ln 2}{t} \sum_{m=1}^{N} V_{m} \frac{\partial c^{*}\left(\mathbf{x}, s_{m}\right)}{\partial x_{1}} \\
\frac{\partial c(\mathbf{x}, t)}{\partial x_{2}} & \simeq \frac{\ln 2}{t} \sum_{m=1}^{N} V_{m} \frac{\partial c^{*}\left(\mathbf{x}, s_{m}\right)}{\partial x_{2}}
\end{aligned}
$$

where

$$
\begin{aligned}
s_{m} & =\frac{\ln 2}{t} m \\
V_{m} & =(-1)^{\frac{N}{2}+m} \sum_{k=\left[\frac{m+1}{2}\right]}^{\min \left(m, \frac{N}{2}\right)} \frac{k^{N / 2}(2 k) !}{\left(\frac{N}{2}-k\right) ! k !(k-1) !(m-k) !(2 k-m) !}
\end{aligned}
$$

A simple script has been developed to calculate the values of the coefficients $V_{m}, m=1,2, \ldots, N$ for any number $N$. Table (1) shows the values of $V_{m}$ for $N=4,6,8,10$.

\section{Numerical results}

In order to justify the analysis derived in the previous sections, we will consider several problems either as test examples of analytical solutions or problems without simple analytical solutions. We assume each problem belongs to a system which is valid in given spatial and time domains and governed by equation (1.1) and satisfying the initial condition (2.2) and some boundary conditions as defined in Section 2. The characteristics of the system which are represented by the coefficients $d_{i j}(\mathbf{x}), v_{i}(\mathbf{x}), \alpha(\mathbf{x})$ in equation (1.1) are assumed to be of the form (3.1), (3.2) and (3.3) in which $g(\mathbf{x})$ is an exponential function of the form (3.4). The coefficients 
$d_{i j}(\mathbf{x}), v_{i}(\mathbf{x}), \alpha(\mathbf{x})$ represents respectively the diffusivity or conductivity, the velocity of flow existing in the system and the change rate of the unknown or dependent variable $c(\mathbf{x}, t)$. As the flow is assumed to be incompressible, the velocity $v_{i}(\mathbf{x})$ must satisfy $\partial v_{i}(\mathbf{x}) / \partial x_{i}=0$.

A standard BEM is derived by discretizing the boundary integral equation (3.18). The boundary is discretized into a number of elements and the dependent variables $c$ and $P$ are assumed to be constant on each element (constant element), taking their values on the mid-point of each element. For the contour integrals, we use the 10-nodal-point Bode quadrature of error of order $O\left(h^{11}\right)$ where $9 h$ is equal to the length of the element. To some extent, this quadrature provides avoidance of the computation of the values of the fundamental solutions (3.16) and (3.17) on their singularities (if available), as the sink points never coincide with the source points (mid-point of elements). It also will guarantee the convergence of the solution as the number of boundary elements is increased.

The value of $N$ in (3.19) for the Stehfest formula is chosen to be $N=10$. For each time $t$, a number of $N=10$ solutions of Laplace transform frame $c^{*}$ and its derivatives are calculated. Then, we use the values of these solutions to find the solutions of the original frame $c$ and its derivatives by utilizing the Stehfest formula (3.19).

For a simplicity, a unit square (depicted in Figure 1) will be taken as the geometrical domain for all problems. A number of 320 elements of equal length, namely 80 elements on each side of the unit square, are used. A FORTRAN script is developed to compute the solutions.

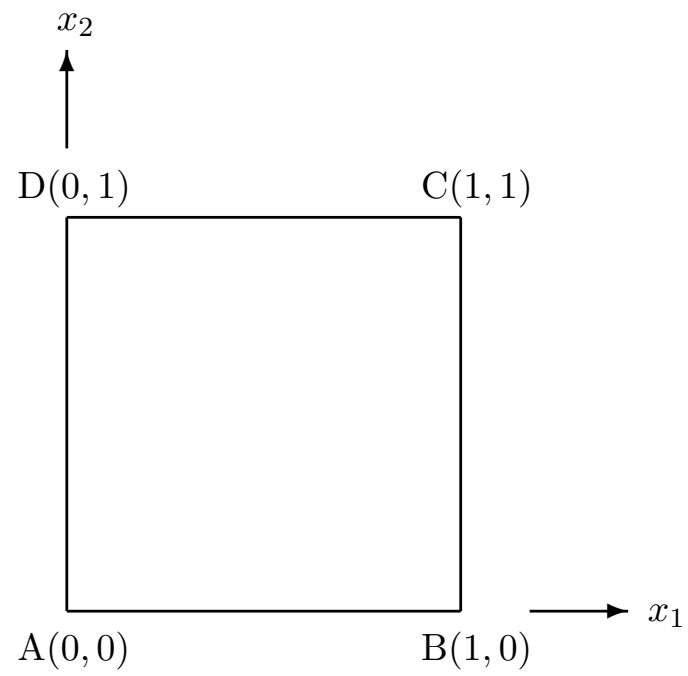

Figure 1: The domain $\Omega$

\subsection{Test problems}

Other aspects that will be justified are the accuracy and consistency (between the scattering and flow) of the numerical solutions. The errors of the numerical solutions are computed using the formula $\sqrt{\left(\mu_{a}^{2}-\mu_{n}^{2}\right)}$ where $\mu_{a}, \mu_{n}$ are the analytical and numerical solutions, respectively. The analytical solutions are assumed to take a separable variables form

$$
c(\mathbf{x}, t)=g^{-1 / 2}(\mathbf{x}) h(\mathbf{x}) f(t)
$$




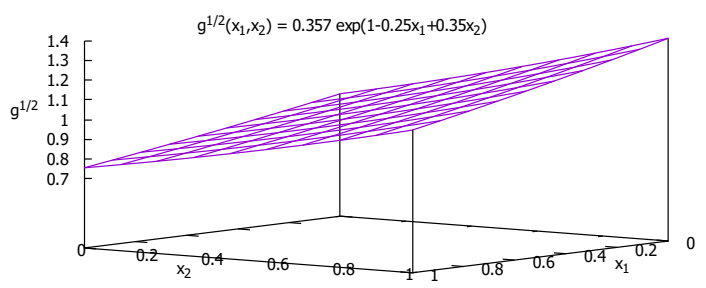

Figure 2: Function $g(\mathbf{x})$

where

$$
h(\mathbf{x})=2.7 \exp \left[-\left(1+0.1 x_{1}+0.15 x_{2}\right)\right]
$$

The function $g^{1 / 2}(\mathbf{x})$ is

$$
g^{1 / 2}(\mathbf{x})=0.357 \exp \left(1-0.25 x_{1}+0.35 x_{2}\right)
$$

and depicted in Figure 2.

We will consider three forms of time variation functions $f(t)$ of time domain $t=[0: 5]$ which are

$$
\begin{aligned}
& f(t)=1-\exp (-1.5 t) \\
& f(t)=0.2 t \\
& f(t)=t(5-t) / 6.25
\end{aligned}
$$

We take mutual coefficients $\hat{d}_{i j}$ and $\hat{v}_{i}$ for the problems

$$
\hat{d}_{i j}=\left[\begin{array}{cc}
1 & 0.15 \\
0.15 & 0.45
\end{array}\right] \quad \hat{v}_{i}=(0.7,0.5)
$$

so that from (3.5) we have

$$
\lambda=0.091375
$$

For $h(\mathbf{x})$ to be a solution to (3.14), the value of $\hat{\alpha}$ has to be

$$
\hat{\alpha}=0.07825 / s
$$

We also take a set of boundary conditions (see Figure 1) 


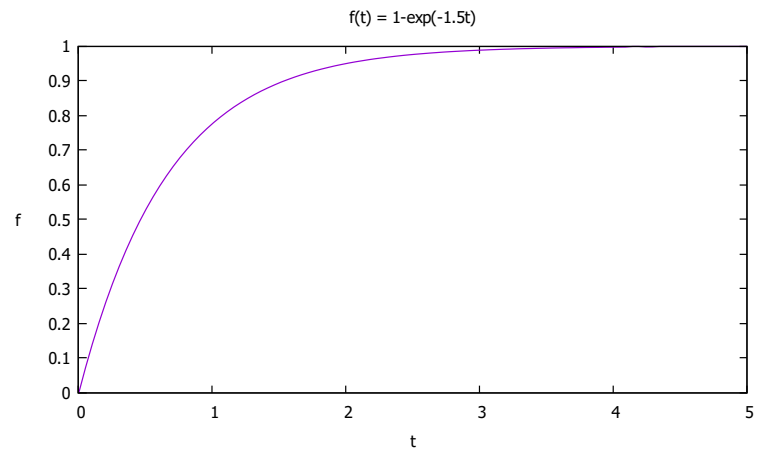

Figure 3: Function $f(t)$ for Problem 1
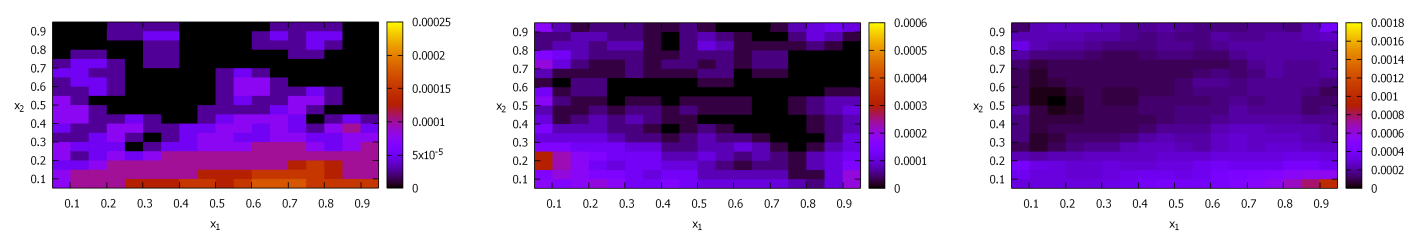

Figure 4: The errors of solutions $c$ (left), $\partial c / \partial x_{1}$ (middle), $\partial c / \partial x_{2}$ (right) at $t=2.5$ for Problem 1

Problem 1: First, we suppose that the time variation function is

$$
f(t)=1-\exp (-1.5 t)
$$

Function $f(t)$ is depicted in Figure 3 .

Figure 4 and Table 2 show the accuracy of the BEM solutions. The errors occur in the fourth decimal place for the $c$ and the derivatives $\partial c / \partial x_{1}$ and $\partial c / \partial x_{2}$ solutions. Figure 5 shows the consistency between the scattering and the flow solutions which verifies that the solutions for the derivatives had also been computed correctly. Figure 6 shows that the solution $c$ changes with time $t$ in a similar way the function $f(t)=1-\exp (-1.5 t)$ does (see Figure 3 ) and tends to approach a steady state solution as the time goes to infinity, as expected.
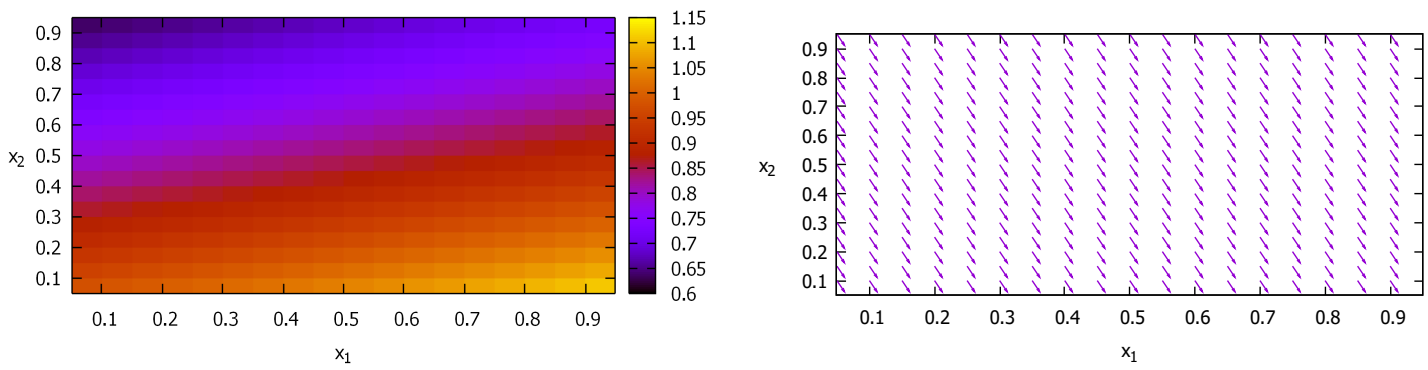

Figure 5: Solutions $c$ and $\left(\partial c / \partial x_{1}, \partial c / \partial x_{2}\right)$ at $t=2.5$ for Problem 1 
Table 2: A comparison between numerical and analytical solutions at the centre point $(0.5,0.5)$ for Problem 1

\begin{tabular}{|c|c|c|c|c|c|c|}
\hline \multirow{2}{*}{$t$} & \multicolumn{2}{|c|}{ Numerical solution } & \multicolumn{3}{c|}{ Analytical solution } \\
\cline { 2 - 7 } & $c$ & $\frac{\partial c}{\partial x_{1}}$ & $\frac{\partial c}{\partial x_{2}}$ & $c$ & $\frac{\partial c}{\partial x_{1}}$ & $\frac{\partial c}{\partial x_{2}}$ \\
\hline 0.0005 & 0.0006 & 0.0001 & -0.0003 & 0.0006 & 0.0001 & -0.0003 \\
\hline 0.5 & 0.4535 & 0.0680 & -0.2267 & 0.4534 & 0.0680 & -0.2267 \\
\hline 1.0 & 0.6676 & 0.1001 & -0.3337 & 0.6675 & 0.1001 & -0.3338 \\
\hline 1.5 & 0.7685 & 0.1153 & -0.3841 & 0.7687 & 0.1153 & -0.3843 \\
\hline 2.0 & 0.8162 & 0.1224 & -0.4080 & 0.8164 & 0.1225 & -0.4082 \\
\hline 2.5 & 0.8390 & 0.1258 & -0.4194 & 0.8390 & 0.1259 & -0.4195 \\
\hline 3.0 & 0.8499 & 0.1275 & -0.4248 & 0.8497 & 0.1275 & -0.4248 \\
\hline 3.5 & 0.8552 & 0.1283 & -0.4275 & 0.8547 & 0.1282 & -0.4274 \\
\hline 4.0 & 0.8577 & 0.1286 & -0.4287 & 0.8571 & 0.1286 & -0.4285 \\
\hline 4.5 & 0.8588 & 0.1288 & -0.4293 & 0.8582 & 0.1287 & -0.4291 \\
\hline 5.0 & 0.8593 & 0.1289 & -0.4295 & 0.8587 & 0.1288 & -0.4294 \\
\hline
\end{tabular}

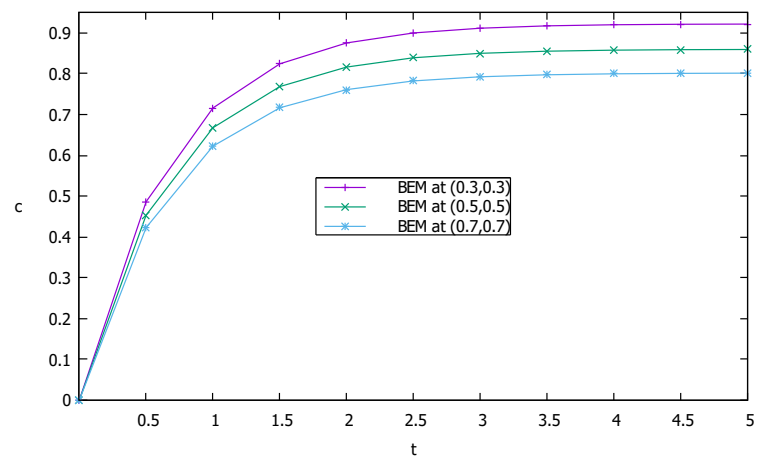

Figure 6: Solutions $c$ for Problem 1 


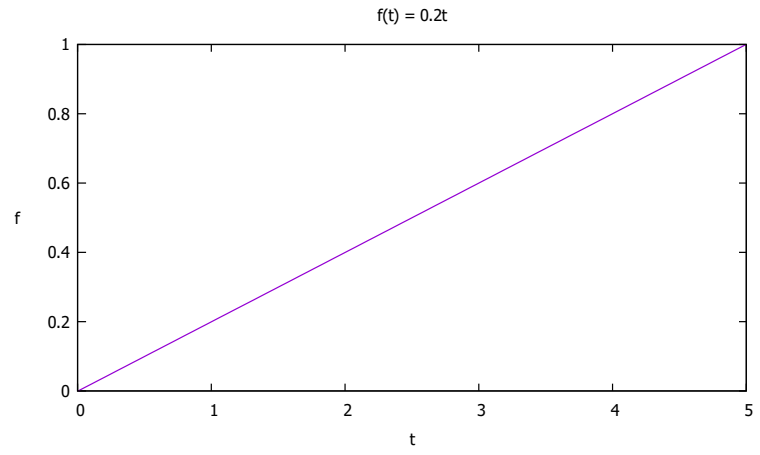

Figure 7: Function $f(t)$ for Problem 2
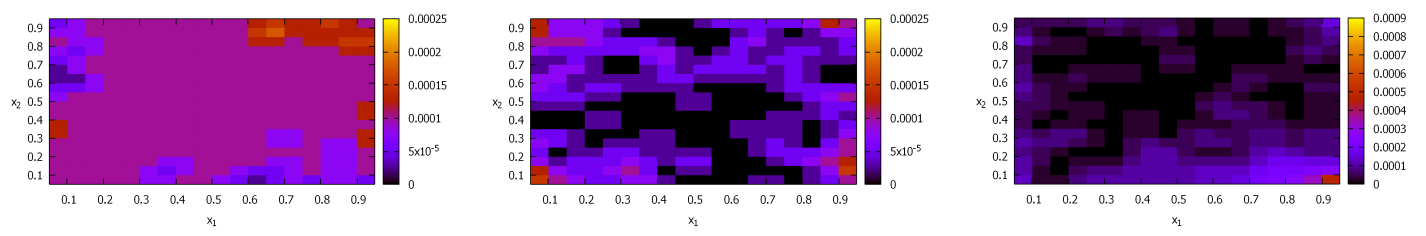

Figure 8: The errors of solutions $c$ (left), $\partial c / \partial x_{1}$ (middle), $\partial c / \partial x_{2}$ (right) at $t=2.5$ for Problem 2

Problem 2: Next, we suppose that the time variation function is (see Figure 7)

$$
f(t)=0.2 t
$$

Figure 8 and Table 3 show the accuracy of the BEM solutions. The errors occur in the fourth decimal place for the $c$ and the derivatives $\partial c / \partial x_{1}$ and $\partial c / \partial x_{2}$ solutions. Figure 9 shows the consistency between the scattering and the flow solutions. Figure 10 shows that the solution $c$ changes with time $t$ in a manner which is almost similar to as the function $f(t)=0.2 t$ does (see Figure 7), as expected.

Problem 3: Now, we suppose that the time variation function is (see Figure 11)
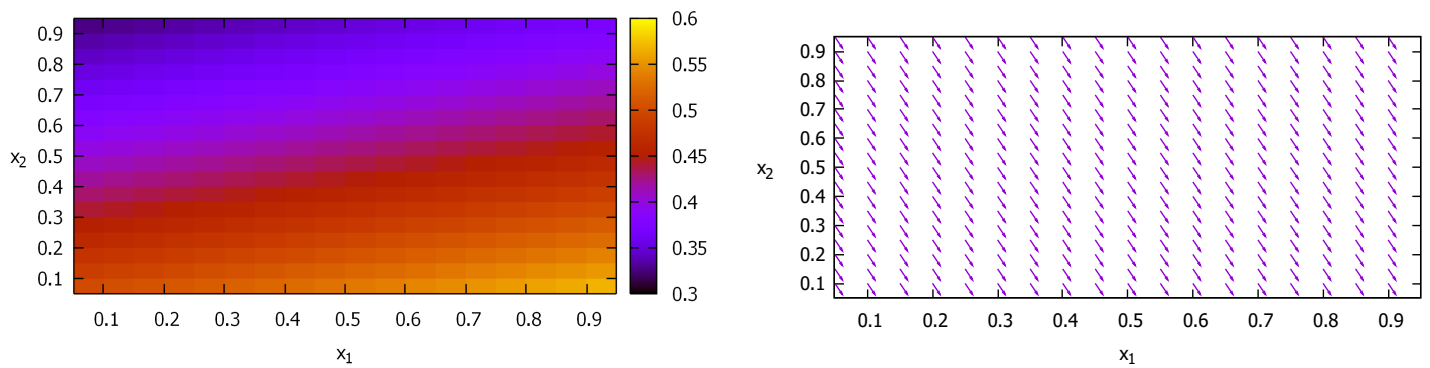

Figure 9: Solutions $c$ and $\left(\partial c / \partial x_{1}, \partial c / \partial x_{2}\right)$ at $t=2.5$ for Problem 2 
Table 3: A comparison between numerical and analytical solutions at the centre point $(0.5,0.5)$ for Problem 2

\begin{tabular}{|c|c|c|c|c|c|c|}
\hline \multirow{2}{*}{$t$} & \multicolumn{2}{|c|}{ Numerical solution } & \multicolumn{3}{c|}{ Analytical solution } \\
\cline { 2 - 7 } & $c$ & $\frac{\partial c}{\partial x_{1}}$ & $\frac{\partial c}{\partial x_{2}}$ & $c$ & $\frac{\partial c}{\partial x_{1}}$ & $\frac{\partial c}{\partial x_{2}}$ \\
\hline 0.0005 & 0.0001 & 0.0000 & -0.0000 & 0.0001 & 0.0000 & -0.0000 \\
\hline 0.5 & 0.0859 & 0.0129 & -0.0430 & 0.0859 & 0.0129 & -0.0430 \\
\hline 1.0 & 0.1719 & 0.0258 & -0.0859 & 0.1718 & 0.0258 & -0.0859 \\
\hline 1.5 & 0.2578 & 0.0387 & -0.1289 & 0.2578 & 0.0387 & -0.1289 \\
\hline 2.0 & 0.3438 & 0.0516 & -0.1718 & 0.3437 & 0.0516 & -0.1718 \\
\hline 2.5 & 0.4297 & 0.0645 & -0.2148 & 0.4296 & 0.0644 & -0.2148 \\
\hline 3.0 & 0.5157 & 0.0773 & -0.2578 & 0.5155 & 0.0773 & -0.2578 \\
\hline 3.5 & 0.6016 & 0.0902 & -0.3007 & 0.6015 & 0.0902 & -0.3007 \\
\hline 4.0 & 0.6875 & 0.1031 & -0.3437 & 0.6874 & 0.1031 & -0.3437 \\
\hline 4.5 & 0.7735 & 0.1160 & -0.3866 & 0.7733 & 0.1160 & -0.3866 \\
\hline 5.0 & 0.8594 & 0.1289 & -0.4296 & 0.8592 & 0.1289 & -0.4296 \\
\hline
\end{tabular}

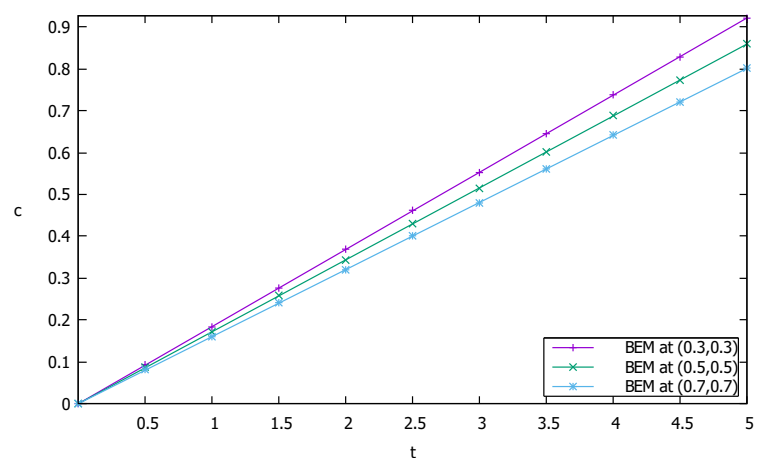

Figure 10: Solutions $c$ for Problem 2 


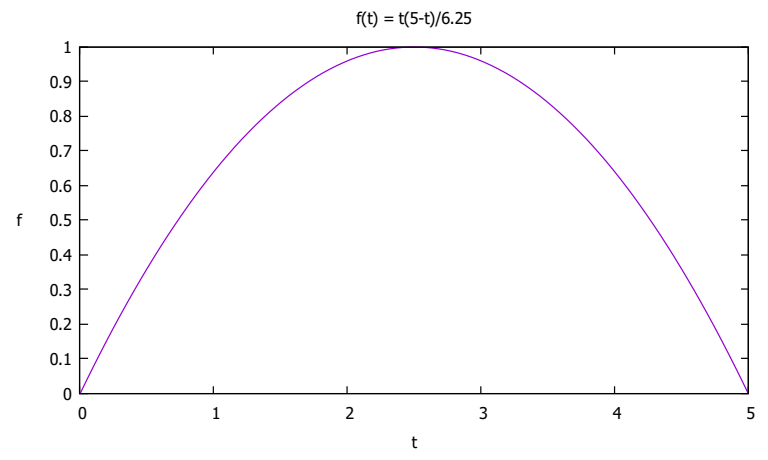

Figure 11: Function $f(t)$ for Problem 3
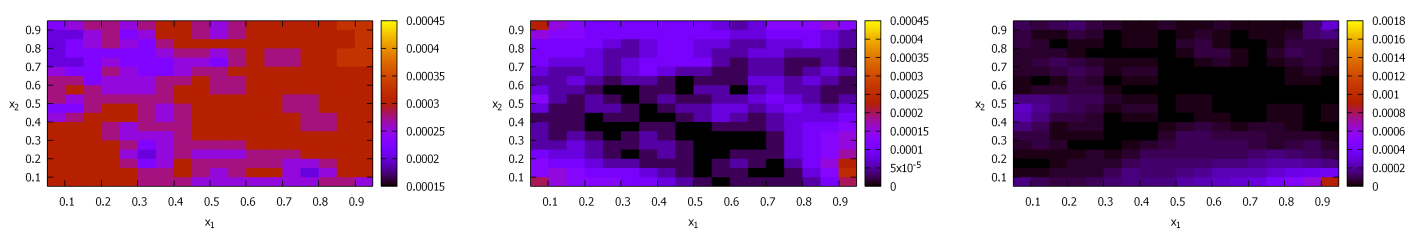

Figure 12: The errors of solutions $c$ (left), $\partial c / \partial x_{1}$ (middle), $\partial c / \partial x_{2}$ (right) at $t=2.5$ for Problem 3

$$
f(t)=t(5-t) / 6.25
$$

Figure 12 and Table 4 show the accuracy of the BEM solutions. Again, the errors occur in the fourth decimal place for the $c$ and the derivatives $\partial c / \partial x_{1}$ and $\partial c / \partial x_{2}$ solutions. Figure 13 shows the consistency between the scattering and the flow solutions which again verifies that the solutions for the derivatives had also been computed correctly. Figure 14 shows that the solution $c$ changes with time $t$ in a similar way the function $f(t)=t(5-t) / 6.25$ does.
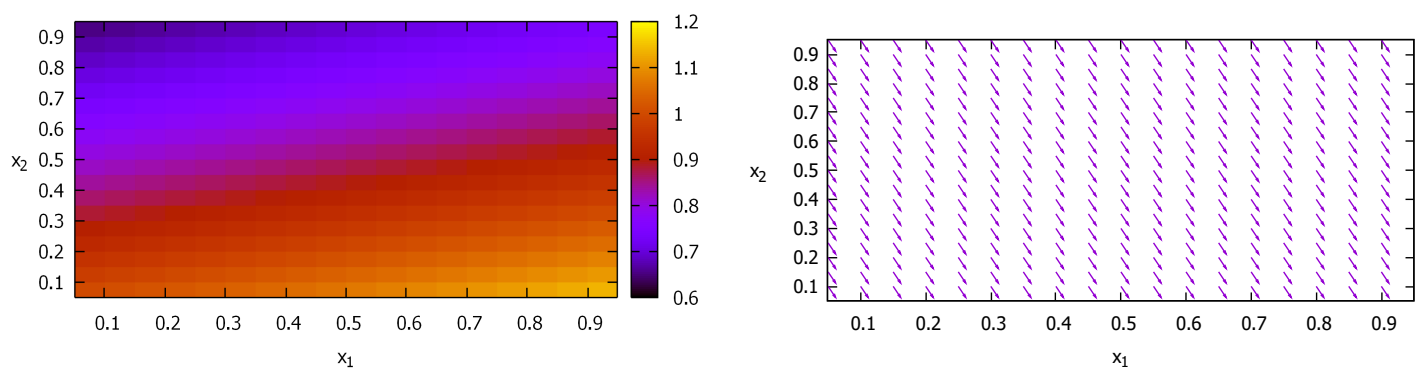

Figure 13: Solutions $c$ and $\left(\partial c / \partial x_{1}, \partial c / \partial x_{2}\right)$ at $t=2.5$ for Problem 3 
Table 4: A comparison between numerical and analytical solutions at the centre point $(0.5,0.5)$ for Problem 3

\begin{tabular}{|c|c|c|c|c|c|c|}
\hline \multirow{2}{*}{$t$} & \multicolumn{3}{|c|}{ Numerical solution } & \multicolumn{3}{c|}{ Analytical solution } \\
\cline { 2 - 7 } & $c$ & $\frac{\partial c}{\partial x_{1}}$ & $\frac{\partial c}{\partial x_{2}}$ & $c$ & $\frac{\partial c}{\partial x_{1}}$ & $\frac{\partial c}{\partial x_{2}}$ \\
\hline 0.0005 & 0.0003 & 0.0001 & -0.0002 & 0.0003 & 0.0001 & -0.0002 \\
\hline 0.5 & 0.3094 & 0.0464 & -0.1547 & 0.3093 & 0.0464 & -0.1547 \\
\hline 1.0 & 0.5500 & 0.0825 & -0.2749 & 0.5499 & 0.0825 & -0.2750 \\
\hline 1.5 & 0.7219 & 0.1083 & -0.3609 & 0.7217 & 0.1083 & -0.3609 \\
\hline 2.0 & 0.8251 & 0.1238 & -0.4124 & 0.8249 & 0.1237 & -0.4124 \\
\hline 2.5 & 0.8595 & 0.1289 & -0.4296 & 0.8592 & 0.1289 & -0.4296 \\
\hline 3.0 & 0.8252 & 0.1238 & -0.4124 & 0.8249 & 0.1237 & -0.4124 \\
\hline 3.5 & 0.7221 & 0.1083 & -0.3609 & 0.7217 & 0.1083 & -0.3609 \\
\hline 4.0 & 0.5502 & 0.0825 & -0.2750 & 0.5499 & 0.0825 & -0.2750 \\
\hline 4.5 & 0.3096 & 0.0464 & -0.1548 & 0.3093 & 0.0464 & -0.1547 \\
\hline 5.0 & 0.0003 & 0.0000 & -0.0002 & 0.0000 & 0.0000 & -0.0000 \\
\hline
\end{tabular}

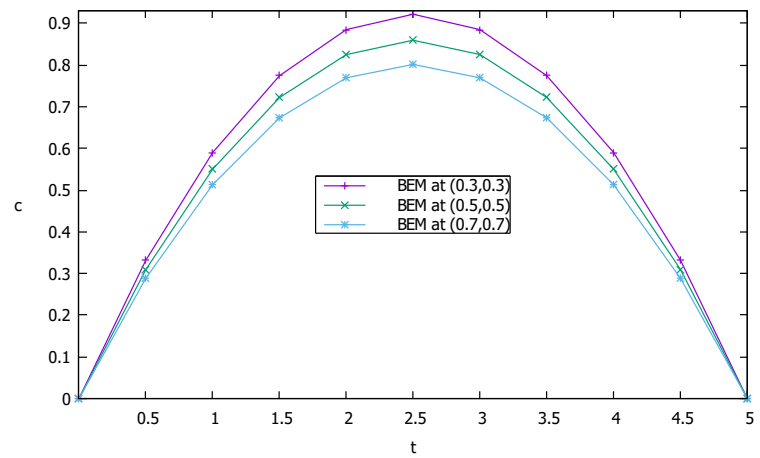

Figure 14: Solutions $c$ for Problem 3 


\subsection{Examples without analytical solutions}

Furthermore, we will show the impact of the anisotropy and the inhomogeneity of the material under consideration on the solutions. We choose

$$
\hat{v}_{i}=(0.7,0.5) \quad \hat{\alpha}=1
$$

Problem 4: For this problem the medium is supposed to be inhomogeneous or homogeneous, anisotropic or isotropic with grading function $g(\mathbf{x})$, constant coefficients $\hat{d}_{i j}$ and corresponding $\lambda$ satisfying (3.5) and (3.6) as respectively follows:

- inhomogeneous and anisotropic case

$$
\begin{aligned}
g^{1 / 2}(\mathbf{x}) & =0.357 \exp \left(1-0.25 x_{1}+0.35 x_{2}\right) \\
\hat{d}_{i j} & =\left[\begin{array}{cc}
1 & 0.15 \\
0.15 & 0.45
\end{array}\right] \\
\lambda & =0.091375
\end{aligned}
$$

- inhomogeneous and isotropic case

$$
\begin{aligned}
g^{1 / 2}(\mathbf{x}) & =0.357 \exp \left(1-0.25 x_{1}+0.35 x_{2}\right) \\
\hat{d}_{i j} & =\left[\begin{array}{ll}
1 & 0 \\
0 & 1
\end{array}\right] \\
\lambda & =0.185
\end{aligned}
$$

- homogeneous and isotropic case

$$
\begin{aligned}
g^{1 / 2}(\mathbf{x}) & =1 \\
\hat{d}_{i j} & =\left[\begin{array}{ll}
1 & 0 \\
0 & 1
\end{array}\right] \\
\lambda & =0
\end{aligned}
$$

- homogeneous and anisotropic case

$$
\begin{aligned}
g^{1 / 2}(\mathbf{x}) & =1 \\
\hat{d}_{i j} & =\left[\begin{array}{cc}
1 & 0.15 \\
0.15 & 0.45
\end{array}\right] \\
\lambda & =0
\end{aligned}
$$

The boundary conditions are that (see Figure 1)

$$
\begin{aligned}
& P=0 \text { on side } \mathrm{AB} \\
& c=0 \text { on side } \mathrm{BC} \\
& P=0 \text { on side } \mathrm{CD} \\
& P=1 \text { on side } \mathrm{AD}
\end{aligned}
$$

There is no simple analytical solution for the problem. The results in Figure 15 show that the solutions $c$ at point $(0.5,0.1)$ coincide with the solutions at $(0.5,0.9)$ only when the material is isotropic homogeneous. This is to be expected as the system is in fact geometrically symmetric 

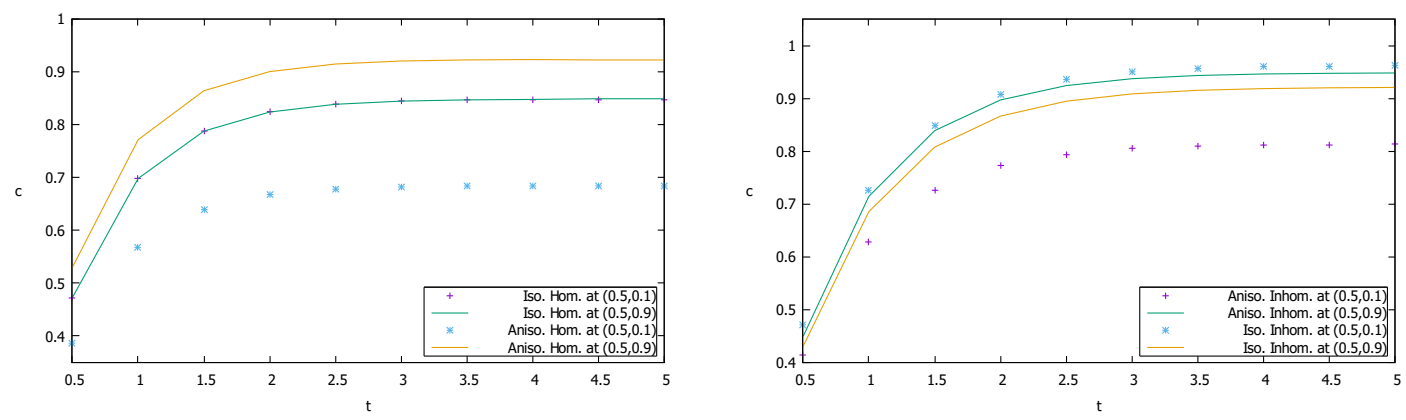

Figure 15: Symmetry of solutions $c$ about $x_{2}=0.5$ for Problem 4

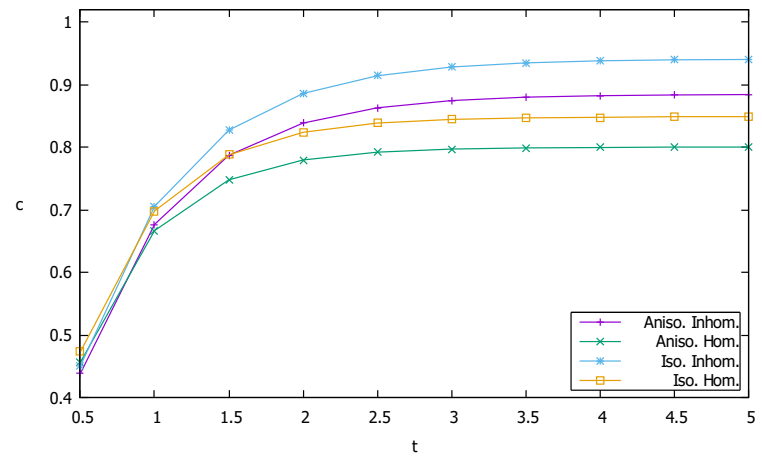

Figure 16: Solutions $c$ at $\left(x_{1}, x_{2}\right)=(0.5,0.5)$ for Problem 4

about the axis $x_{2}=0.5$ if the material is isotropic homogeneous. Otherwise, if the material is anisotropic or inhomogeneous, the solutions $c$ at point $(0.5,0.1)$ do not coincide with the solutions at $(0.5,0.9)$. This indicates that anisotropy and inhomogeneity give impact to the values of solution $c$ for being asymmetric about $x_{2}=0.5$. Furthermore, the results in Figure 16 also indicate the impact of the anisotropy and inhomogeneity of the material. The values of $c$ differ for each type of anisotropy and inhomogeneity.

Problem 5: We consider the inhomogeneous and anisotropic case of Problem 4 again. But we change slightly the set of the boundary conditions of Problem 4 especially on the side AD. Now we use three cases of the boundary condition on the side AD, namely

$$
\begin{aligned}
& P=1-\exp (-1.5 t) \text { on side } \mathrm{AD} \\
& P=0.2 t \text { on side } \mathrm{AD} \\
& P=t(5-t) / 6.25 \text { on side } \mathrm{AD}
\end{aligned}
$$

The results in Figure 17 are expected. The trends of the solutions $c$ mimics the trends of the exponential function $1-\exp (-1.5 t)$, the linear function $0.2 t$ and the quadratic function $t(5-t) / 6.25$ of the boundary condition on side AD. Specifically, for the exponential function $1-\exp (-1.5 t)$, as time $t$ goes to infinity, values of this function go to 1 . So for big value of $t$, 


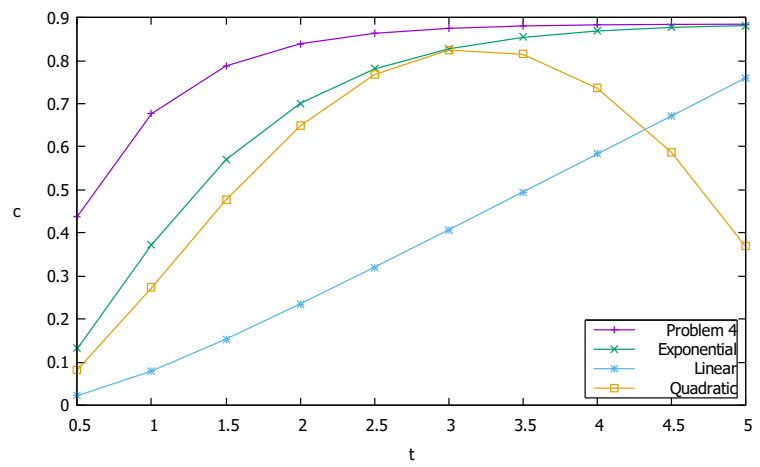

Figure 17: Solutions $c$ at $\left(x_{1}, x_{2}\right)=(0.5,0.5)$ for Problem 5

Problem 5 is similar to Problem 4 of the anisotropic inhomogeneous case. And the two plots of solutions $c$ for Problem 4 and Problem 5 in Figure 17 verifies this, they approach a same steady state solution as $t$ gets bigger.

\section{Conclusion}

A mixed Laplace transform and standard BEM has been used to find numerical solutions to initial boundary value problems for anisotropic functionally graded materials which are governed by the diffusion-convection equation (1.1) of incompressible flow. The method is easy to be implemented and involves a time variable free fundamental solution therefore it gives more accurate solutions. It does not generate round errors as it solves the boundary integral equation (3.18) independently for each specific value of $t$ at which the solution is computed. Unlikely, the methods with time variable fundamental solution may produce less accurate solutions as the fundamental solution sometimes contain time singular points and also solution for the next time step is based on the solution of the previous time step so that the round error may propagate.

The numerical method has been applied to exponentially graded materials. As the coefficients $d_{i j}(\mathbf{x}), v_{i}(\mathbf{x}), \alpha(\mathbf{x})$ do depend on the spatial variable $\mathbf{x}$ only and on the same inhomogeneity or grading function $g(\mathbf{x})$, it will be good to extend the study in the future to the case when the coefficients depend on different grading functions varying also with the time variable $t$.

In order to use the boundary integral equation (3.18), the values $c(\mathbf{x}, t)$ or $P(\mathbf{x}, t)$ of the boundary conditions as stated in Section (2) of the original system in time variable $t$ have to be Laplace transformed first. This means that from the beginning when we set up a problem, we actually put a set of approximating boundary conditions. Therefore it is really important to find a very accurate technique of numerical Laplace transform inversion.

\section{Acknowledgments}

The author would like to thank Hasanuddin University and Kementerian Pendidikan, Kebudayaan, Riset, dan Teknologi of Indonesia for the provided support. 


\section{References}

[1] M. A. H. Assagaf, A. Massinai, A. Ribal, S. Toaha S, M. I. Azis, Numerical simulation for steady anisotropic-diffusion convection problems of compressible flow in exponentially graded media, Journal of Physics: Conference Series, 1341(8) (2019), 082016.

[2] M. I. Azis, BEM solutions to exponentially variable coefficient Helmholtz equation of anisotropic media, Journal of Physics: Conference Series, 1277 (2019), 012036.

[3] M. I. Azis, Numerical solutions for the Helmholtz boundary value problems of anisotropic homogeneous media, Journal of Physics: Conference Series, 381 (2019), 42-51.

[4] M. I. Azis, Standard-BEM solutions to two types of anisotropic-diffusion convection reaction equations with variable coefficients, Engineering Analysis with Boundary Elements, 105 (2019), 87-93.

[5] M. I. Azis, I. Solekhudin I, M. H. Aswad, A. R. Jalil, Numerical simulation of twodimensional modified Helmholtz problems for anisotropic functionally graded materials, Journal of King Saud University - Science, 32(3) (2020), 2096-2102.

[6] S. Baja, S. Arif, Fahruddin, N. Haedar, M. I. Azis, Boundary element method solutions for steady anisotropic-diffusion convection problems of incompressible flow in quadratically graded media, Journal of Physics: Conference Series, 1341(8) (2019), 062019.

[7] Fendoğlu H, Bozkaya C, Tezer-Sezgin M, DBEM and DRBEM solutions to 2D transient convection-diffusion-reaction type equations, Engineering Analysis with Boundary Elements, 93 (2018), 124-134.

[8] A. Haddade, M. I. Azis, Z. Djafar, St. N. Jabir, B. Nurwahyu, Numerical solutions to a class of scalar elliptic BVPs for anisotropic, IOP Conf. Ser.: Earth Environ. Sci., 279 (2019), 012007 .

[9] A. Haddade, E. Syamsuddin, M. F. I. Massinai, M. I. Azis, A. I. Latunra, Numerical solutions for anisotropic-diffusion convection problems of incompressible flow in exponentially graded media, Journal of Physics: Conference Series, 1341(8) (2019), 082015.

[10] S. Hamzah, M. I. Azis, A. Haddade, A. K. Amir, Numerical solutions to anisotropic BVPs for quadratically graded media governed by a Helmholtz equation, IOP Conf. Ser.: Mater. Sci. Eng., 619 (2019), 012060.

[11] S. Hamzah, A. Haddade, A. Galsan, M. I. Azis, A. M. Abdal, Numerical solution to diffusion convection-reaction equation with trigonometrically variable coefficients of incompressible flow, Journal of Physics: Conference Series, 1341(8) (2019), 082005.

[12] Hernandez-Martinez E, Puebla H, Valdes-Parada F, Alvarez-Ramirez J, Nonstandard finite difference schemes based on Green's function formulations for reaction-diffusion-convection systems, Chemical Engineering Science, 94 (2013), 245-255.

[13] St. N. Jabir, M. I. Azis, Z. Djafar, B. Nurwahyu, BEM solutions to a class of elliptic BVPs for anisotropic trigonometrically graded media, IOP Conference Series: Materials Science and Engineering, 619 (2019), 012059. 
[14] A. R. Jalil, M. I. Azis, S. Amir, M. Bahri, S. Hamzah, Numerical simulation for anisotropicdiffusion convection reaction problems of inhomogeneous media, Journal of Physics: Conference Series, 1341(8) (2019), 082013.

[15] Khaeruddin, A. Galsan, M. I. Azis, N. Ilyas, Paharuddin, Boundary value problems governed by Helmholtz equation for anisotropic trigonometrically graded materials: A boundary element method solution, Journal of Physics: Conference Series, 1341 (2019), 062007.

[16] N. Lanafie, N. Ilyas, M. I. Azis, A. K. Amir, A class of variable coefficient elliptic equations solved using BEM, IOP Conference Series: Materials Science and Engineering, 619 (2019), 012025 .

[17] N. Lanafie, P. Taba, A. I. Latunra, Fahruddin, M. I. Azis, On the derivation of a boundary element method for diffusion convection-reaction problems of compressible flow in exponentially inhomogeneous media, Journal of Physics: Conference Series, 1341(6) (2019), 062013.

[18] Q. Li, Z. Chai, B. Shi, Lattice Boltzmann model for a class of convection-diffusion equations with variable coefficients, Computers and Mathematics with Applications, 70 (2015), 548 561.

[19] M. Meenal, T. I. Eldho, Two-dimensional contaminant transport modeling using mesh free point collocation method (PCM), Engineering Analysis with Boundary Elements, 36 (2012), $551-561$.

[20] B. Nurwahyu, B. Abdullah, A. Massinai, M. I. Azis, Numerical solutions for BVPs governed by a Helmholtz equation of anisotropic FGM, IOP Conference Series: Earth and Environmental Science, 279 (2019), 012008.

[21] Paharuddin, Sakka, P. Taba, S. Toaha, M. I. Azis, Numerical solutions to Helmholtz equation of anisotropic functionally graded materials, Journal of Physics: Conference Series, 1341 (2019), 082012.

[22] R. Pettres, L. A. de Lacerda, Numerical analysis of an advective diffusion domain coupled with a diffusive heat source, Engineering Analysis with Boundary Elements, 84 (2017), 129-140.

[23] A. Rap, L. Elliott, D. B. Ingham, D. Lesnic, X. Wen, DRBEM for Cauchy convectiondiffusion problems with variable coefficients, Engineering Analysis with Boundary Elements, 28 (2004), 1321-1333.

[24] N. Rauf, H. Halide, A. Haddade, D. A. Suriamihardja, M. I. Azis, A numerical study on the effect of the material's anisotropy in diffusion convection reaction problems, Journal of Physics: Conference Series, 1341(8) (2019), 082014.

[25] J. Ravnik, L. Škerget, A gradient free integral equation for diffusion-convection equation with variable coefficient and velocity, Engineering Analysis with Boundary Elements, 37 (2013), 683-690.

[26] J. Ravnik, L. Škerget, Integral equation formulation of an unsteady diffusion-convection equation with variable coefficient and velocity, Computers and Mathematics with Applications, 66 (2014), 2477-2488. 
[27] I. Raya, Firdaus, M. I. Azis, Siswanto, A. R. Jalil, Diffusion convection-reaction equation in exponentially graded media of incompressible flow: Boundary element method solutions, Journal of Physics: Conference Series, 1341(8) (2019), 082004.

[28] Sakka, E. Syamsuddin, B. Abdullah, M. I. Azis, A. M. A. Siddik, On the derivation of a boundary element method for steady anisotropic-diffusion convection problems of incompressible flow in trigonometrically graded media, Journal of Physics: Conference Series, 1341(8) (2019), 062020.

[29] N. Salam, A. Haddade, D. L. Clements, M. I. Azis, A boundary element method for a class of elliptic boundary value problems of functionally graded media, Engineering Analysis with Boundary Elements, 84 (2017), 186-190.

[30] N. Salam, D. A. Suriamihardja, D. Tahir, M. I. Azis, E. S. Rusdi, A boundary element method for anisotropic-diffusion convection-reaction equation in quadratically graded media of incompressible flow, Journal of Physics: Conference Series, 1341(8) (2019), 082003.

[31] S. Suryani, J. Kusuma, N. Ilyas, M. Bahri, M. I. Azis, A boundary element method solution to spatially variable coefficients diffusion convection equation of anisotropic media, Journal of Physics: Conference Series, 1341(6) (2019), 062018.

[32] R. Syam, Fahruddin, M. I. Azis, A. Hayat, Numerical solutions to anisotropic FGM BVPs governed by the modified Helmholtz type equation, IOP Conference Series: Materials Science and Engineering, 619(1) (2019), 012061.

[33] F. Wang, W. Chen, A. Tadeu, C. G. Correia, Singular boundary method for transient convection-diffusion problems with time-dependent fundamental solution, International Journal of Heat and Mass Transfer, 114 (2017), 1126-1134.

[34] X-H. Wu, Z-J. Chang, Y-L. Lu, W-Q. Tao, S-P. Shen, An analysis of the convectiondiffusion problems using meshless and mesh based methods, Engineering Analysis with Boundary Elements, 36 (2012), 1040-1048.

[35] H. Yoshida, M. Nagaoka, Multiple-relaxation-time lattice Boltzmann model for the convection and anisotropic diffusion equation, Journal of Computational Physics, 229 (2010), $7774-7795$.

[36] C. Zoppou, J. H. Knight, Analytical solution of a spatially variable coefficient advectiondiffusion equation in up to three dimensions, Applied Mathematical Modelling, 23 (1999), $667-685$.

Mohammad Ivan Azis Department of Mathematics, Hasanuddin University, Makassar, Indonesia

E-mail: ivan@unhas.ac.id 